\title{
Soluções Otimizadas para o Problema de Localização de Máxima Cobertura em Redes Militarizadas 4G/LTE
}

\author{
Emerson de O. Antunes ${ }^{1}$, Marcos F. Caetano ${ }^{1}$, Marcelo A. Marotta ${ }^{1}$, \\ Aleteia Araujo $^{1}$, Lucas Bondan ${ }^{1,2}$, Rodolfo I. Meneguette ${ }^{3}$, e \\ Geraldo P. Rocha Filho ${ }^{1}$
}

\author{
${ }^{1}$ Departamento de Ciência da Computação, Universidade de Brasília - UnB \\ ${ }^{2}$ Rede Nacional de Ensino e Pesquisa - RNP \\ ${ }^{3}$ Instituto de Ciências Matemáticas e de Computação - Universidade de São Paulo USP \\ emerson.antunes@aluno.unb.br, \{mfcaetano, marcelo.marotta\}@unb.br, \\ aleteia@unb.br, lucas.bondanernp.br, meneguettedicmc.usp.br, \\ geraldofeunb.br
}

\begin{abstract}
Resumo. Este trabalho propõe resolver o problema de localização de máxima cobertura do Centro de Coordenação de Operações Móvel (CCOp Mv) que visa apoiar o comando operacional do Exército Brasileiro. Este problema consiste em selecionar, em uma região limitada e com infraestrutura precária de comunicação na área de operação das tropas terrestres, as posições dos veículos equipados com antenas para maximizar a área de cobertura. Em razão disso, foi proposta uma modelagem analítica baseada em Problemas Lineares Inteiros Mistos que fundamentou duas soluções de otimização: (i) EALLOCATOR - Exact ALLOCATiOn seRvice; e (ii) M-ALLOCATOR - Metaheuristic ALLOCATiOn seRvice. As soluções foram avaliadas em um cenário que emprega o uso do CCOp Mv em apoio à uma operação de resgate com base na tragédia ocorrida em janeiro de 2019 em Brumadinho-MG. Os resultados mostraram que o E-ALLOCATOR é adequado quando há baixa carga de trabalho, enquanto o M-ALLOCATOR é adequado para os cenários com alta carga de trabalho. Além disso, os resultados indicam que o M-ALLOCATOR fornece soluções subótimas dentro de um tempo computacional adequado para todas as instâncias do problema.
\end{abstract}

Abstract. This work proposes to solve the maximal covering location problem of the Mobile Operations Coordination Center (CCOp Mv), which aims to support the operational command of the Brazilian Army. This problem consists of selecting, in a limited region and with poor communication infrastructure to the ground troops's operating area, the positions of vehicles equipped with antennas that maximize the coverage area. For this reason, analytical modeling based on the mixed-integer linear problem was proposed that guided two optimization solutions: (i) E-ALLOCATOR - Exact ALLOCATiOn seRvice; and (ii) M-ALLOCATOR - Metaheuristic ALLOCATiOn seRvice. The solutions were evaluated in a scenario that employs CCOp Mv to support a rescue operation based on the tragedy that occurred in January 2019 in Brumadinho-MG. The results showed that E-ALLOCATOR is suitable for a low workload on the network, while M-ALLOCATOR is suitable for scenarios with a high workload. Furthermore, the results indicate that M-ALLOCATOR provides almost optimal solutions within the adequate computational time for all instances of the problem. 


\section{Introdução}

A localização das estações rádio base (BTS, do inglês Base Transceiver Station) além de ser de suma importância para o desempenho das redes de rádio celulares, é uma das etapas que compõe o complexo processo de planejamento deste sistema de comunicação [Mathar and Niessen 2000]. Tal processo envolve três etapas, sendo elas: (i) o uso de modelos de predição de propagação; (ii) descrição analítica do tráfego demandado pelo serviço; e (iii) localização e configurações das BTSs. O uso de modelos de predição de propagação serve para o cálculo da área de cobertura baseada em modelos empíricos de medições de energia do espectro de Rádio Frequência (RF) [Rappaport et al. 1996]. Por outro lado, a descrição analítica do tráfego demandado por um serviço contempla a carga de trabalho a ser suportada pelo sistema de comunicação sem fio, definindo requisitos de métricas de desempenho a serem cumpridos, como capacidade entregada mínima e latência máxima com os terminais de comunicação. Por fim, a localização das BTSs define o posicionamento geográfico levando em consideração a instalação de todos os elementos necessários, tais como mastros, antenas e a própria BTS. Assim sendo, devido à complexidade deste processo, diversos trabalhos foram propostos na área dos Sistemas de Apoio a Decisão (SAD), como forma de automatizar o processamento das informações necessárias para o melhor posicionamento das BTSs.

No contexto de SAD, destaca-se a idealização do Centro de Coordenação de Operações Móvel (CCOp Mv) que visa a criação de um centro de comando e controle para apoiar o comando operacional do Exército Brasileiro. O CCOp Mv possui como elemento principal os nós de acesso, que são viaturas militares dotadas de diferentes tipos de enlaces de comunicação. Dentre as tecnologias de comunicação disponíveis, prevalece o sistema de telefonia celular militarizado 4G/LTE (LTE, do inglês Long Term Evolution). É importante destacar que não é um tarefa trivial definir o melhor posicionamento geográfico no terreno dessas viaturas, de forma a permitir uma cobertura adequada para a realização de operações militares que necessitam de comunicação 4G/LTE. Esta tarefa enquadra-se no planejamento do melhor posicionamento das BTSs e, em específico, é tratada como uma derivação do problema de localização de máxima cobertura (MCLP, do inglês Maximal Covering Location Problem) [Church and ReVelle 1974]. O MCLP é um problema NP-Completo cujo objetivo visa a criação de uma área de cobertura para atender o maior número de pontos de demanda.

Diferentes SADs foram propostos para lidar com o problema do MCLP. Na maior parte desses estudos [Mehboob et al. 2016, Atta et al. 2018, Bagherinejad and Shoeib 2018, Seda et al. 2020], as soluções propostas sugerem melhorias para lidar com MCLP de maneira genérica, i.e., apesar de existir abordagens que exploram aplicações do MCLP para domínio específico, como posicionamento de garagens de veículos [Gazani and Niaki 2021], alocação de antenas [Costa 2016] ou até mesmo veículos de comunicação [Yang et al. 2020] aplicados em operações de resgate, tais abordagens tratam apenas de um subconjunto do problema, não refletindo a modelagem para cenários reais. A modelagem de [Costa 2016] é simplificada e seus resultados são avaliados apenas em termos de velocidade. As abordagens de [Costa 2016] e [Yang et al. 2020] não exploram os ajustes dos hiperparâmetros dos algoritmos meta-heurísticos empregados. Como citado em [Farahani et al. 2012] e ratificado por [Alizadeh et al. 2021], os modelos desenvolvidos estão longe dos problemas do mundo real, indicando um campo de pesquisa promissor que este trabalho investiga. 
Diante dessas limitações, este trabalho propõe resolver o problema de localização dos nós de acesso do CCOp Mv, que são as BTSs do seu sistema de comunicação. Para isso, é apresentada a proposta de um modelo analítico baseado em Problemas Lineares Inteiros Mistos (PLIM). Tal modelo serviu de base para a proposta de duas soluções de otimização, sendo elas: (i) E-ALLOCATOR - Exact ALLOCATiOn seRvice - solução exata; e (ii) M-ALLOCATOR - Metaheuristic ALLOCATiOn seRvice - solução metaheurística. Para validar ambas soluções, foi modelado um cenário que ilustra o uso do CCOp Mv em apoio à uma operação de resgate, baseado na tragédia ocorrida em janeiro de 2019 em Brumadinho - MG. Resultados evidenciam que o E-ALLOCATOR é a solução adequada quando há baixa carga de trabalho, enquanto o M-ALLOCATOR é a solução adequada para os cenários com alta carga de trabalho.

O restante deste artigo está estruturado da seguinte maneira. A Seção 2 apresenta os trabalhos relacionados com foco no MCLP. A Seção 3 apresenta a estratégia adotada para modelar as soluções propostas. A Seção 4 apresenta os resultados para validar as soluções propostas. Por fim, a Seção 5 apresenta as conclusões e os trabalhos futuros.

\section{Trabalhos Relacionados}

Esta seção apresenta as abordagens que exploram o problema do MCLP no contexto de $\mathrm{SAD}$, além de identificar as limitações encontradas em tais abordagens às quais esta pesquisa investiga. Salienta-se que o MCLP foi inicialmente explorado por [Church and ReVelle 1974], resultando no desenvolvimento de modelos voltados para a cobertura do maior número de pontos de demanda com um número mínimo de BTS. Atualmente, o MCLP vem sendo investigado adicionando-se novas características ao problema, como energia de transmissão, perda de sinal, tempo de processamento, bem como o uso de novos algoritmos heurísticos e meta-heurísticos para solucionar o problema [Mehboob et al. 2016, Yang et al. 2020, Alizadeh et al. 2021].

Em [Costa 2016] o autor propõe o uso de algoritmo memético para a resolução do problema de localização de antenas. O trabalho aborda detalhes como o cálculo do alcance das antenas, a perda de sinal por degradação da distância, entre outros. Ao tentar a generalização do problema, sua solução acaba não explorando questões relevantes, tais como: o tempo de processamento e convergência da sua solução, bem como a qualidade dos resultados obtidos em comparação com outros trabalhos, diferente desta pesquisa.

Já em [Atta et al. 2018] é proposta uma abordagem com base na utilização de Algoritmo Genético (AG) que realiza o refinamento local da solução para a convergência mais rápida na resolução do MCLP. Tal abordagem consiste em aplicar alterações nos cromossomos do AG, por meio de um processo de clusterização, para determinar a localização e o número de BTS, de forma a permitir a cobertura de todos os clientes. Enquanto em [Bagherinejad and Shoeib 2018] tratam o problema de localização de cobertura máxima em multi-períodos. A sua solução é baseada no uso de algoritmos de AG e colônia de abelhas. Contudo, as abordagens citadas se limitam a um subconjunto do problema, não explorando as etapas já existentes nos algoritmos genéticos tradicionais.

Na obra [Seda et al. 2020] os autores propuseram dois modelos matemáticos, para a resolução do MCLP, no contexto de redes de sensores sem fio com suporte às redes 5G. Os modelos propostos abordam o problema de área de cobertura considerando a infraestrutura de rede pré-existente, restringindo assim sua aplicação a cenários específicos e associados às redes 5G. Os autores em [Gazani and Niaki 2021] exploram o 
problema de posicionamento MCLP considerando equipamentos com diferentes custos. Os autores propõem uma solução exata baseada no solver CPLEX, bem como propõem soluções utilizando algoritmos heurísticos e meta-heurísticos. Os autores utilizam algoritmos genéticos considerando a probabilidade de cruzamento, probabilidade de mutação e taxa de elitismo, utilizando discretização para um subconjunto do MCLP.

Diferente dos trabalhos mencionados, esta pesquisa diferencia-se por considerar um cenário de infraestrutura de comunicação inexistente, provendo um modelo analítico baseado no PLIM que fundamentou a proposta de duas soluções otimizadas, as quais fornecem subsídios para a construção do módulo de otimização do sistema de apoio à decisão proposto do CCOp Mv, como apresentado a seguir.

\section{Serviço de Apoio à Decisão do CCOp Mv}

Esta seção apresenta um serviço de apoio à decisão do CCOp Mv para tratar do problema de posicionamento dos BTSs em regiões onde a infraestrutura de comunicação é precária, ou mesmo inexistente, sob a ótica de uma rede 4G/LTE. Para tanto, foram modeladas duas soluções otimizadas para lidar com o problema MCLP no contexto do CCOp Mv, sendo elas: (i) E-ALLOCATOR que é uma solução exata com base em PLIM; e (ii) MALLOCATOR que é uma solução meta-heurística de natureza combinatória com base em um AG. O serviço proposto tem como objetivo principal cobrir o maior número de pontos de demanda com o menor número de nós de acesso, ao mesmo tempo que provê uma maior área de apoio nas operações que necessitam de comunicação crítica.

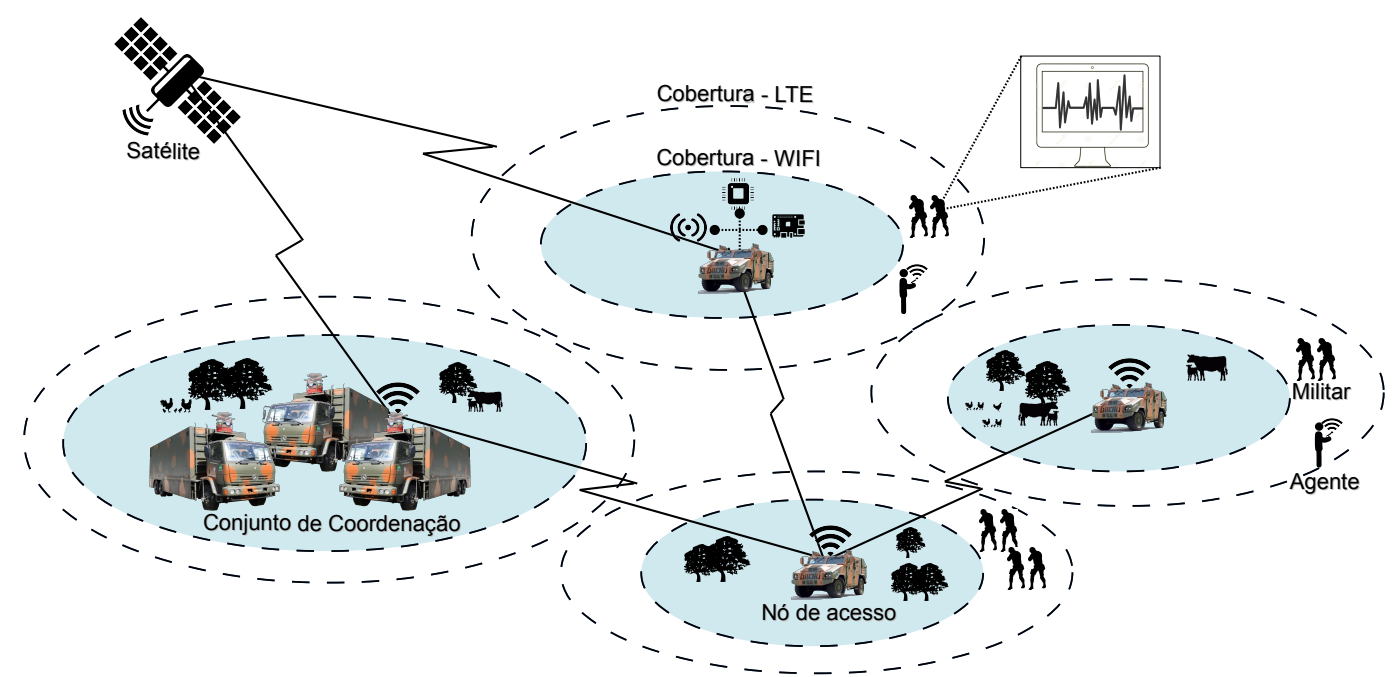

Figura 1. Cenário de funcionamento do serviço de apoio à decisão do CCOp Mv.

Na Figura 1 é apresentado o cenário de funcionamento do serviço proposto no processo de alocação dos veículos/BTSs. A idealização desta proposta surgiu a partir do CCOp Mv, cujo objetivo é prover os meios de comando e controle necessários para coordenação das operações do Exército Brasileiro. Trata-se de um projeto do Programa Proteger ${ }^{1}$ destinado a suprir a Força Terrestre da infraestrutura de TIC necessária ao apoio das operações terrestres em ambientes desprovidos de infraestrutura de comunicações. $\mathrm{O}$ CCOp Mv é composto por 5 (cinco) caminhões de 5 toneladas que formam o Conjunto

\footnotetext{
${ }^{1}$ http://www.epex.eb.mil.br/index.php/proteger
} 
de Coordenação das operações e por até 8 veículos de 2 e $\frac{1}{2}$ toneladas denominados nós de acesso. Os veículos do Conjunto de Coordenação operam juntos e, portanto, neste trabalho são considerados como ocupantes de uma única posição de alocação. Todos estes veículos disponibilizam uma plataforma de comunicação formada pelas seguintes tecnologias: sistema de telefonia militarizado 4G/LTE; rádios UHF, VHF e HF; Sistema Rádio Digital Troncalizado (SRDT); equipamentos de TI; equipamentos com acesso ao Sistema Militar de Comunicações por Satélite e Sistemas de Comando e Controle de Consciência Situacional e Apoio à Decisão. Desta forma, as soluções apresentadas neste trabalho para o problema de posicionamento das BTSs, vão além da questão científica na resolução do problema, passando pela sua aplicação prática no escopo do CCOP Mv.

Para resolver o problema de alocação das BTSs do CCOp Mv, dividiu-se a atuação do sistema proposto em duas etapas: (i) pré-processamento; e (ii) otimização. Na etapa de pré-processamento, as matrizes de pesos e conectividade são geradas por meio dos dados geográficos do terreno, tais como: latitude, longitude e elevação. Essas informações são utilizadas para determinar a existência ou não de linha de visada entre a posição geográfica dos nós de acesso e os usuários do sistema. Além disso, também são consideradas as características de propagação dos sinais sem fio, interferência, relaçã sinal-ruído (Signal-toNoise Ratio ou SNR), desvanecimento do sinal, potência de transmissão e recepção, bem como seus limiares de aceitação para a definição da efetividade da comunicação de rádio. Nesta etapa, utilizou-se o modelo de propagação de Okumura Hata [Seybold 2005], o qual provê a predição da potência do sinal recebido nas diversas posições do terreno. As informações obtidas com este modelo servem de entrada para a segunda etapa da solução proposta, a fase de otimização. Nesta etapa, as soluções propostas E-ALLOCATOR e MALLOCATOR utilizam as informações de pré-processamento para a definição da quantidade e dos locais onde os nós de acesso devam ser posicionados, de forma a permitir uma maior área de cobertura para o sistema 4G/LTE.

\subsection{Modelo do Serviço}

Nesta subseção é apresentada a modelagem matemática do serviço e a formulação do problema de otimização adotado. O problema foi modelado a partir da associação dos usuários com antenas que serão posicionadas em diferentes posições geográficas. A Tabela 1 apresenta as notações usadas na modelagem e na definição do problema.

A modelagem é iniciada pela definição do conjunto $\mathcal{M}=\{1,2, \ldots, M\}$ de nós que representam os clientes do sistema de comunicação e do conjunto $\mathcal{N}=\{1,2, \ldots$, $N\}$ de nós que representam as possíveis localidades para instalação das antenas (nós de acesso). Cada par de nós $(m, n)$ compõe uma aresta conectada quando $\{m \in \mathcal{M}$ e $n \in \mathcal{N} \mid$ $\varsigma(m, n) \neq 0\}$, no qual $\varsigma(m, n)$ representa a função de conexão e assume os valores entre 1 e 5 que denotam a potência do sinal recebido, quando o cliente $m$ pode ser atendido pela antena $n$, e 0 caso contrário. A função de conexão pode ser iterada considerando as combinações entre todos os $M$ nós com todos os $N$ nós para gerar a matriz de pesos $P=\{0,1,2,3,4,5\}_{M x N}$, do conjunto de arestas. A partir da matriz de pesos $P$ deriva-se a matriz binária de conectividade $C$ que tem as mesmas dimensões que $P$, porém com valores binários, $C=\{0,1\}_{M x N}$. Seja $p_{m n} \in P$ e $c_{m n} \in C$, esta derivação segue a seguinte regra:

$$
c_{m n}= \begin{cases}1, & \text { se } p_{m n} \neq 0 \\ 0, & \text { se } p_{m n}=0\end{cases}
$$


Tabela 1. Notações usadas na modelagem e definição do problema.

\begin{tabular}{c|c}
\hline Notação & Parâmetro \\
\hline$G(\mathcal{M} \cup \mathcal{N}, P)$ & Grafo do sistema \\
$\mathcal{M}$ & Conjunto de posições de demanda dos clientes \\
$\mathcal{N}$ & Conjunto de localidades para instalação das antenas \\
$\varsigma(m, n)$ & Função de conexão \\
$P$ & Matriz de pesos das arestas ou conjunto de arestas \\
$C$ & Matriz de conectividade (MxN) \\
$\phi$ & Número máximo de antenas \\
$\psi$ & Número máximo de clientes suportados por BTS \\
$x_{m}$ & Variável de decisão do cliente $m$ \\
$y_{n}$ & Variável de decisão da posição de instalação da antena $n$ \\
$G^{\prime}(\mathcal{N}, A)$ & Grafo de interconexão das antenas \\
$A$ & Matriz de conectividade entre as antenas (NxN) \\
$a_{n k}$ & Elemento da matriz de conectividade $A_{N x N}$ entre as antenas (n,k) \\
$\xi$ & Número mínimo de nós de acesso interconectados \\
\hline
\end{tabular}

Portanto, nosso serviço pode ser definido pelo grafo bipartido $G(\mathcal{M} \cup \mathcal{N}, P)$.

O grafo $G^{\prime}(\mathcal{N}, A)$ que modela as conexões entre os nós de acesso é definido de maneira análoga, no entanto os vértices são apenas elementos do conjunto $\mathcal{N}$. Suas arestas também são determinadas pela iteração da função de conexão $\varsigma(n, k)$, para $n$ e $k \in \mathcal{N}$, pelas permutações dos nós do conjunto $N$, gerando assim a matriz de pesos que é convertida na matriz de conectividade entre as antenas $A=\{0,1\}_{N x N}$, com elementos $a_{n k}=\{0,1\}$ de forma similar à conversão de $P$ em $C$ no grafo do sistema.

\subsection{Definição do Problema}

Nesta subseção, o problema de localização dos nós de acesso do CCOp Mv será decidido como uma extensão do MCLP. Para isso, defini-se as variáveis de decisão, as restrições e os objetivos do MCLP para, em seguida, propor um problema de programação linear inteira mista. As variáveis de decisão definidas e seus significados são dados por:

$x_{m}= \begin{cases}1, & \text { se o cliente } m \text { está associado a alguma antena que lhe dê cobertura; } \\ 0, & \text { caso contrário. }\end{cases}$ $y_{n}= \begin{cases}1, & \text { se a posição } n \text { foi escolhida para ter uma antena instalada; } \\ 0, & \text { caso contrário. }\end{cases}$

Para a construção do nosso modelo, foram definidas seis restrições:

- Restrição 1 - Define que para um ponto de demanda $x_{m}$ seja atendido é necessário que ao menos uma antena $y_{n}$ que lhe dê cobertura seja instalada:

$$
\sum_{n \in \mathcal{N}} y_{n} \cdot c_{m n} \geq x_{m} \quad, \quad \forall m \in \mathcal{M}
$$

onde $c_{m n}$ terá o valor de 1 , caso a antena na posição $y_{n}$ conseguir atender o usuário em $x_{m}$.

- Restrição 2 - Cada antena $(n)$ consegue atender uma quantidade máxima de usuários $(\psi)$ sendo associados a ela $\left(\sum_{m \in \mathbf{M}} x_{m} \cdot c_{m n}\right)$. A quantidade máxima de usuários é um parâmetro específico da antena e pode variar significativamente de 
um fabricante para outro. Além disso, esse parâmetro é influenciado pela banda de operação do eNB, pelo fator de utilização esperado e pela capacidade de downlink e uplink desejadas para cada usuário.

$$
\sum_{m \in \mathcal{M}} x_{m} \cdot c_{m n} \leq \psi \quad, \quad \forall n \in \mathcal{N}
$$

- Restrição 3 - Estabelece que o número de antenas instaladas não deve exceder ao número máximo disponível $(\phi)$.

$$
\sum_{n \in \mathcal{N}} y_{n} \leq \phi
$$

- Restrição 4 - A rede móvel deve estar sempre conectada para prover serviços. Como as antenas são nós móveis, as mesmas devem participar de uma rede entre si para escoamento de dados. Por exemplo, na minuta do projeto CCOp Mv, determina-se que um nó de acesso deve se conectar a pelo menos outros 3 nós para operação correta. Nesse caso, uma antena $(n)$ deve se conectar a no mínimo $(\xi)$ outras antenas $(k)$ caso estas possam ser conectadas $\left(a_{n k}=1\right)$.

$$
\sum_{n \in \mathcal{N}} y_{n} \cdot a_{n k} \geq \xi \quad, \quad \forall k \in \mathcal{N}
$$

- Restrição 5 - Natureza binária da variável aleatória $x_{m}$.

$$
x_{m} \in\{0,1\} \quad, \quad \forall m \in \mathcal{M}
$$

- Restrição 6 - Natureza binária da variável aleatória $y_{n}$.

$$
y_{n} \in\{0,1\} \quad, \quad \forall n \in \mathcal{N}
$$

Diante do exposto, o nosso modelo matemático possui dois objetivos, como apresentado na Equação 7: (i) Minimizar o número de nós de acesso empregados no CCOp Mv para reduzir o desperdício de recursos computacionais e financeiros; e (ii) Maximizar a área de cobertura dos nós de acesso do CCOp Mv, em que $\top\left(p_{m n}\right)$ consiste no maior peso entre as antenas $n$ ativas que atendem ao cliente $m$. Isso é coerente com a realidade, já que os dispositivos se associam à antena que lhe provê o sinal mais forte.

$$
\operatorname{Min} \sum_{n=1}^{N} y_{n}, \quad \operatorname{Max} \sum_{m=1}^{M} \top\left(p_{m n}\right) \cdot x_{m}
$$

\subsection{Soluções Otimizadas}

A modelagem analítica do problema de alocação dos BTSs do CCOp Mv com base no PLIM, serviu de base para as soluções E-ALLOCATOR e M-ALLOCATOR.

\subsubsection{E-ALLOCATOR}

Com o objetivo de percorrer todo o espaço de busca para tratar do problema de posicionamento dos nós de acesso do CCOp Mv, foi proposto o E-ALLOCATOR: uma solução exata com base no algoritmo Branch and Bound (B\&B) para PLIM, como apresentado no Algoritmo 1. Para tanto, o Algoritmo 1 inicia percorrendo o espaço de busca (linhas 
7-25). Em seguida, analisa as soluções que atendam às restrições do modelo apresentadas na Subseção 3.2, excluindo as soluções infactíveis, i.e., que não atendem às restrições (linhas 9 e 10), ou que apresentem resultados inferiores aos já analisados (linhas 12 e 13). Com isso, o método de busca por ponto inteiro, também conhecido como método Simplex é utilizado. Por se tratar de uma variável inteira, os resultados obtidos de maneira relaxada, i.e., $y_{n} \notin Z_{+}$são subdivididos novamente (linhas 15 a 19) e, em seguida o processo é novamente repetido. Nesse caso, a relaxação linear do modelo se dá pelo limitante superior. Para garantir que a melhor solução encontre o menor número de nós de acesso, o processamento anterior é repetido para um número máximo de antenas $A$ (linha 3). Por último, salienta-se que os resultados são atualizados caso sejam encontradas soluções melhores com um número maior de antenas instaladas (linhas 27-32). A solução desenvolvida utilizou o solver CBC-MIP do framework ORTools ${ }^{2}$.

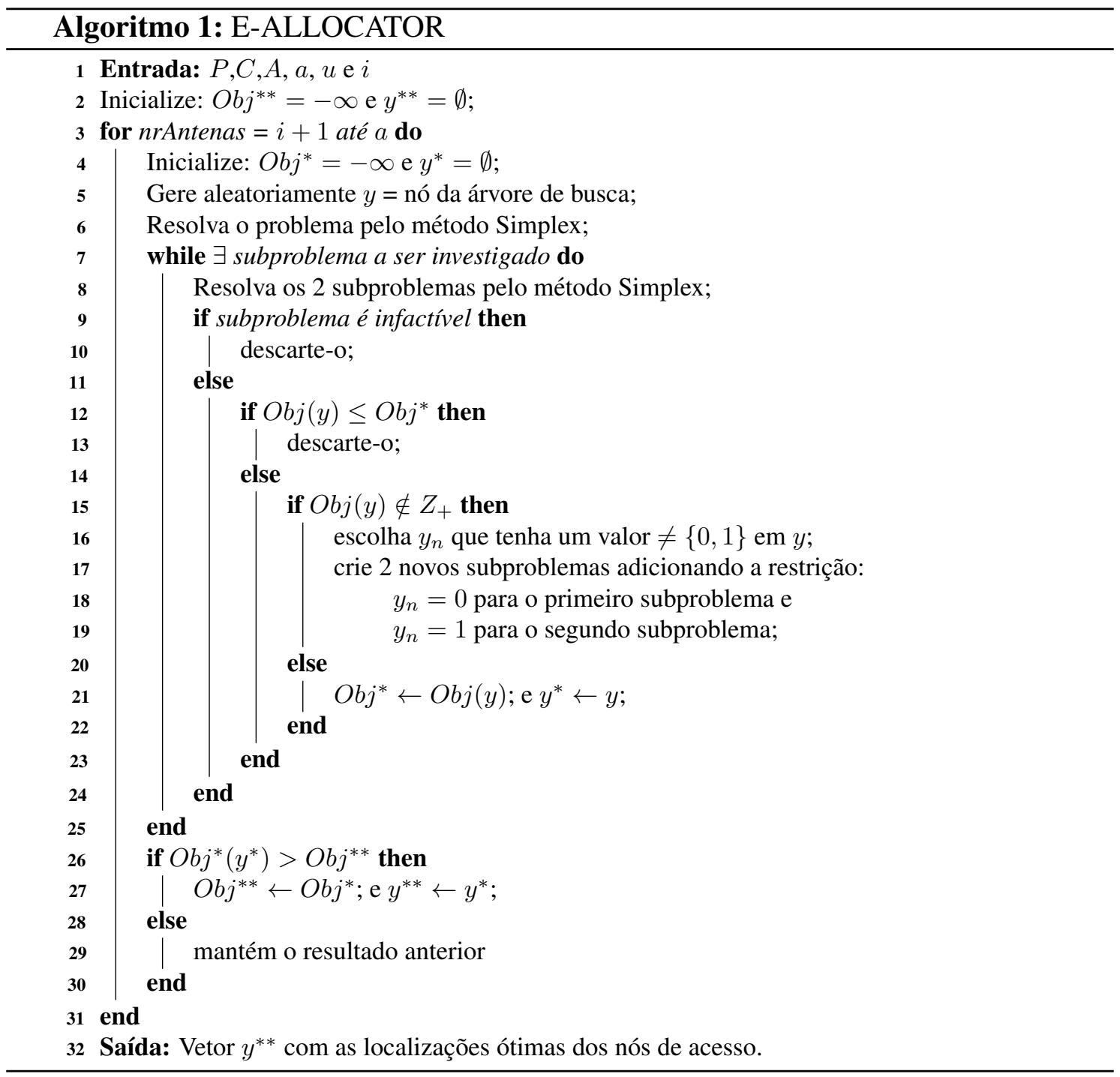

\footnotetext{
${ }^{2}$ https://pypi.org/project/ortools/
} 


\subsubsection{M-ALLOCATOR}

Para tratar os problemas de otimização NP-Completo, as soluções propostas podem ser resolvidas de maneira exata para instâncias cujo o problema é pequeno e que esteja dentro de um período aceitável de tempo. Com o intuito de sanar essas limitações, foi proposto o M-ALLOCATOR que é uma meta-heurística que visa encontrar uma boa solução (sem garantia de ser a ótima) com base em um AG, como apresentado no Algoritmo 2. Para tanto, uma população inicial é instanciada aleatoriamente (linha 3). Em seguida, o grau de adaptação de cada indivíduo é obtido por meio da aplicação da função de aptidão (linha 5). Caso o grau de adaptação do indivíduo mais adaptado encontrado na geração atual seja melhor do que o da geração anterior, os valores da melhor aptidão e o indivíduo melhor adaptado são armazenados para futuras comparações (linhas 6 a 11). Com isso, é realizado o processo evolutivo do $A G$ que são a aplicação dos métodos de seleção, reprodução e mutação para composição da próxima geração (linhas 12 a 15). A solução desenvolvida utilizou a biblioteca PyGAD ${ }^{3} 4$.

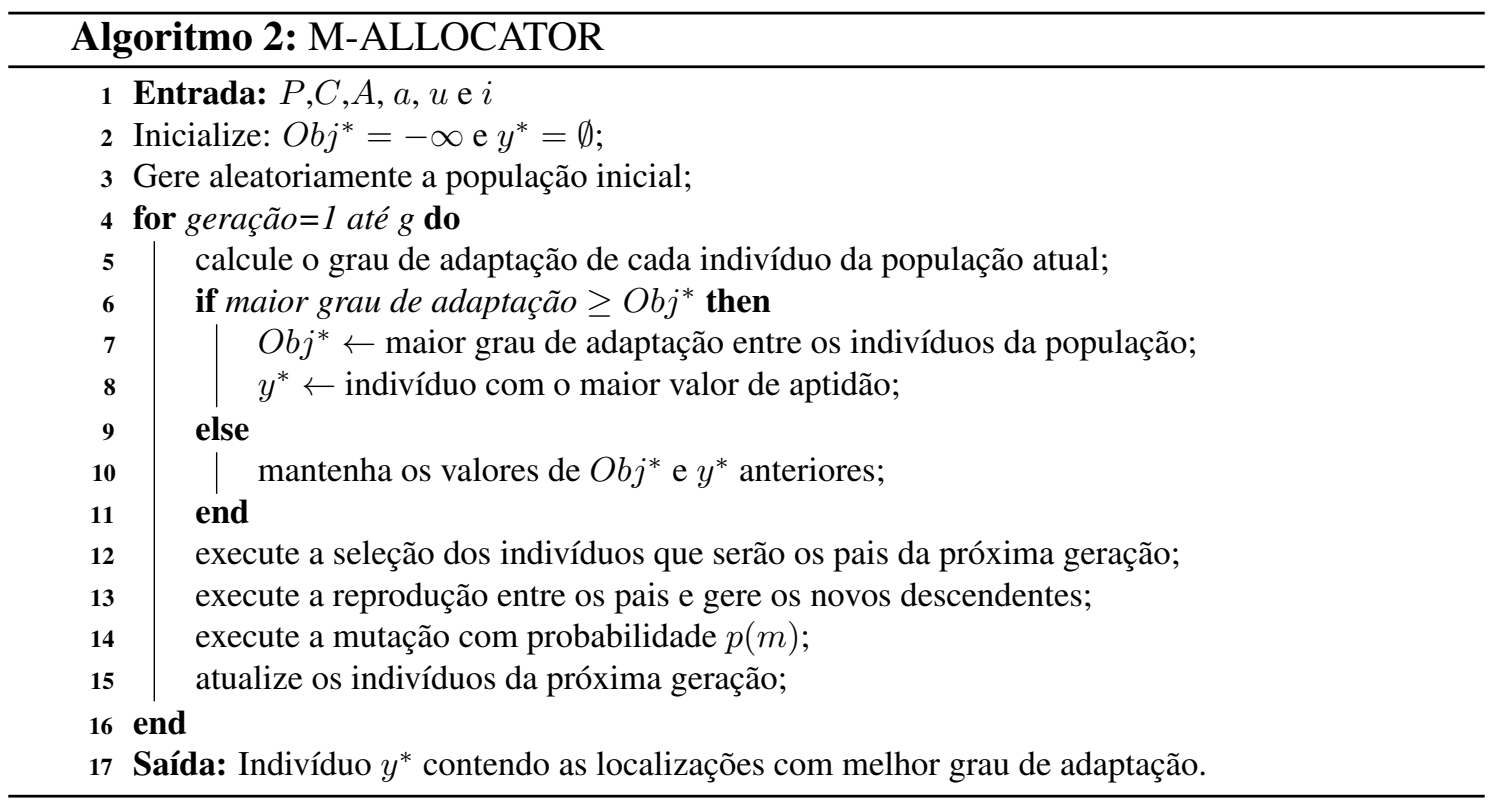

A representação das possíveis soluções do espaço de busca determina a estrutura cromossômica, i.e., a estrutura de um indivíduo. A estrutura proposta nesta pesquisa reflete as posições possíveis das antenas e, portanto, foi constituída de um vetor com $N$ posições ocupadas por genes binários, 0's ou 1's. Cada gene definido como 1 representa a instalação de uma antena na posição desse gene.

A função de aptidão é o mecanismo pelo qual a adaptabilidade de um cromossomo ou indivíduo é avaliada. Neste contexto, a função de aptidão consistiu em uma implementação da função objetivo (Equação 7) e das restrições 1 e 2 modeladas na Subseção 3.2. As restrições 5 e 6 foram garantidas pela natureza binária do gene. Já a Restrição 3 foi implementada por meio de um mecanismo de validação para cada alteração genética, seja na instanciação dos indivíduos da primeira geração, na reprodução ou na mutação. Por último, a Restrição 4 foi garantida por um mecanismo de recompensa para

\footnotetext{
${ }^{3}$ https://pygad.readthedocs.io/en/latest/

${ }^{4}$ https://pypi.org/project/pygad/
} 
as situações que respeitam esta restrição e penalidade para os outros casos.

Para otimizar os hiperparâmetros do AG modelado no M-ALLOCATOR, utilizouse a biblioteca Hyperas ${ }^{5}$. A Tabela 2 apresenta o conjunto de hiperparâmetros utilizados para otimização, nos quais os melhores valores encontrados estão destacado em negrito.

Tabela 2. Hiperparâmetros otimizados.

\begin{tabular}{l|l}
\hline Hiperparâmetro & Valor \\
\hline Probabilidade de cruzamento & {$[0.1 \vdash \mathbf{0 . 7 7} \dashv 1]$} \\
Probabilidade de mutação & {$[0.1 \vdash \mathbf{0 . 7 9} \dashv 1]$} \\
Taxa de elitismo & {$[0.1 \vdash \mathbf{0 . 8 6} \dashv 1]$} \\
Tamanho da população & $\{10,20 \ldots \mathbf{1 1 0} \ldots 200\}$, variando de 10 em 10 \\
Número de gerações & $\{10,11 \ldots \mathbf{7 8} \ldots 100\}$, variando de 1 em 1 \\
Número de pais para reprodução & $\{2,4 \ldots \mathbf{8} \ldots 20\}$, variando de 2 em 2 \\
\hline
\end{tabular}

Além de ajustar os hiperparâmentros, foram avaliados os resultados obtidos pelos métodos de seleção, reprodução e mutação. Na literatura foram identificados seis principais mecanismos de seleção, os quais foram analisados neste trabalho: (i) Estado Estável (EE); (ii) Roleta (R); (iii) Estocástico (E); (iv) Truncamento (Tr); Aleatório (A); e Torneio (To). Relativamente aos métodos de reprodução, foram avaliados os métodos de troca genética por: (i) um Ponto Simples (PS); (ii) Dois Pontos (DP); (iii) Uniforme (U); e (iv) Espalhamento (Es). Já na fase de mutação, foram avaliados os três métodos: (i) Troca (T); (ii) Inversão (I); e (iii) Embaralhamento (Em).

\section{Avaliação de Desempenho}

Nesta seção é avaliado o desempenho das soluções otimizadas para lidar com o MCLP do CCOp Mv.

\subsection{Configurações dos Experimentos}

Para avaliar as soluções propostas foi modelado um cenário que apresenta o uso do CCOp Mv em apoio à uma operação de resgate na qual a infraestrutura de comunicação é inexistente. Tal cenário foi modelado com base na tragédia ocorrida em janeiro de 2019 em Brumadinho - MG. Para definição deste cenário foram distribuídos sobre o mapa da cidade de Brumadinho um conjunto de 200 pontos de demanda (marcadores azuis) e um conjunto de 50 possíveis locais de implantação dos nós de acesso (marcadores pretos), como apresentado na Figura 2. Além da dimensão de 200x50 do cenário de Brumadinho, modelou-se mais dois cenários com as seguintes dimensões 800x400 e 3000x400. Os cenários representam o emprego do Exército Brasileiro em operação de combate no nível companhia, batalhão e brigada, respectivamente. A Tabela 3 apresenta o conjunto de parâmetros utilizados no modelo de propagação de Okumura-Hata [Seybold 2005], em sua versão para ambientes rurais, para gerar as matrizes de pesos e conectividades que são utilizadas como entrada no processo de otimização para gerar os resultados.

É importante salientar que os valores da função de conexão $\varsigma(m, n)$ foram definidos a partir dos valores representativos do gráfico de intensidade do sinal de um smartphone Samsung S10 que possui uma atena de recepção de 0dBi, definidos em [dip ].

\footnotetext{
${ }^{5}$ https://github.com/maxpumperla/hyperas
} 


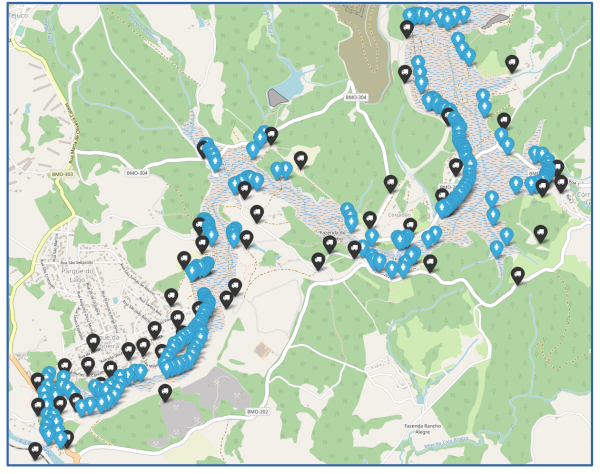

Figura 2. Cenário modelado para o CCOp Mv com base na tragédia de Brumadinho.
Tabela 3. Parâmetros utilizados no cenário modelado.

\begin{tabular}{l|l}
\hline Parâmetro & Valor padrão \\
\hline Altura da antena de transmissão & $10 \mathrm{~m}$ \\
Altura das antenas de recepção & $1,5 \mathrm{~m}$ \\
Potência de transmissão & $25 \mathrm{dBm}$ \\
Ganho da antena de transmissão & $13 \mathrm{dBi}$ \\
Frequência do canal & $700 \mathrm{MHz}$ \\
Dimensão dos cenários & $200 \times 50,800 \times 400 \mathrm{e}$ \\
& $3000 \times 400$ \\
Raio de cobertura $^{1}$ & $2 \mathrm{Km}$ \\
Execuções $^{1}$ Requisito Operacional do projeto CCOp Mv.
\end{tabular}

Assim a potência do sinal predito pelo modelo de propagação foi discretizada em 6 níveis para atribuição à matriz de pesos conforme a seguir:

$$
\varsigma(m, n)= \begin{cases}5, & \text { se a potência recebida } \geq-85 \mathrm{dBm} ; \\ 4, & \text { se a potência recebida } \geq-90 \mathrm{dBm} ; \\ 3, & \text { se a potência recebida } \geq-95 \mathrm{dBm} ; \\ 2, & \text { se a potência recebida } \geq-100 \mathrm{dBm} ; \\ 1, & \text { se a potência recebida } \geq-105 \mathrm{dBm} ; \\ 0, & \text { caso contrário. }\end{cases}
$$

O tempo de processamento, aptidão e convergência foram as métricas utilizadas para análise dos experimentos. $\mathrm{O}$ tempo de processamento e a convergência revelam a velocidade com que as soluções entregam os resultados sendo fundamental para verificação da adequabilidade das soluções em cenários reais. Enquanto a aptidão expressa a qualidade dos resultados obtidos. Para aferição da velocidade foram avaliados o tempo de processamento de 78 gerações. A convergência foi avaliada pela aptidão fornecida em cada geração. Já para medir a aptidão foi avaliada a porcentagem relativa de desvio (PRD) dada pela Equação 8:

$$
P R D=\frac{(\text { Ótima }- \text { melhor Aptidão })}{\text { Ótima }} * 100 .
$$

Os experimentos foram agrupados por fatores a serem avaliados. Para isso, foram realizados três grupos de testes: (i) com variação dos métodos de seleção; (ii) com variação dos métodos de reprodução; e (iii) com variação dos métodos de mutação. Os experimentos foram executados em uma máquina com as seguintes configurações: Intel i5 3300 3.2GHz, quad-core, com 16 GB RAM DDR3.

\subsection{Impacto dos Resultados Obtidos}

Na Figura 3, é apresentada a avaliação de desempenho das soluções otimizadas com a variação dos métodos de seleção. Utilizando como referência o resultado ótimo que o E-ALLOCATOR obteve, nota-se que o tempo de convergência do M-ALLOCATOR com o método EE possui maior velocidade de aproximação, seguido pelo Tr e To, Figura 3a. 
Isso ocorre pois o EE retira os piores indivíduos e substitui por outros indivíduos gerados, tornando o processo mais adaptado para a lista que compõe os candidatos. Isso é ratificado pela métrica aptidão (Figura 3b) e tempo de processamento (Figura 3c) que além de possuir uma amplitude interquartil menor, possui uma mediana ligeiramente melhor quando comparado com outros métodos de seleção do M-ALLOCATOR.

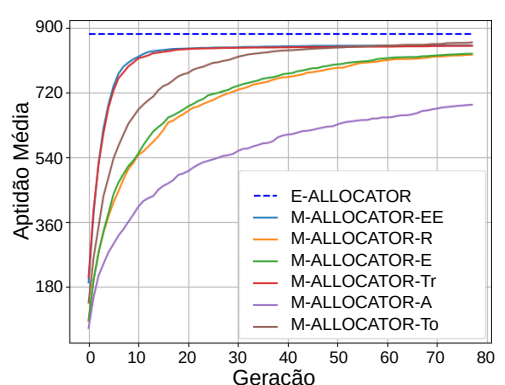

(a) Convergência

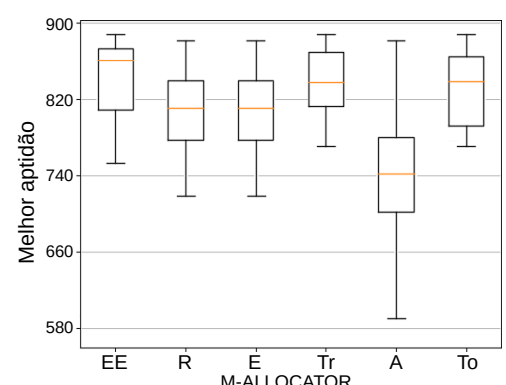

(b) Aptidão



(c) Tempo

Figura 3. Impacto de desempenho com a variação dos métodos de seleção.

Após explorar os métodos de seleção nas soluções otimizadas, explorou-se os métodos de reprodução, como apresentado na Figura 4. Na Figura 4a, observa-se que independente da quantidade de geração, o M-ALLOCATOR com o método DP é o método de reprodução mais eficaz, tendo como base a solução ótima (i.e., E-ALLOCATOR). Isso faz sentido haja vista que o DP explora regiões próximas dos indivíduos, adicionando novas informações a cada geração. Em razão disso, há uma aptidão melhor (Figura 4b) com um menor tempo de processamento (Figura 4c).

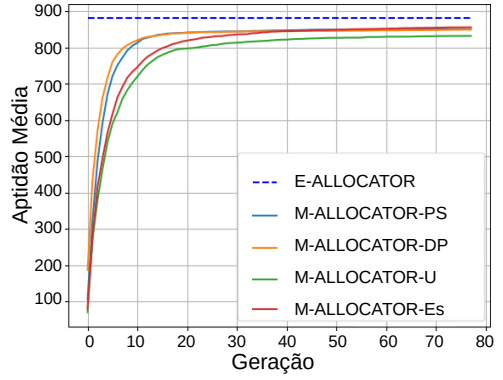

(a) Convergência

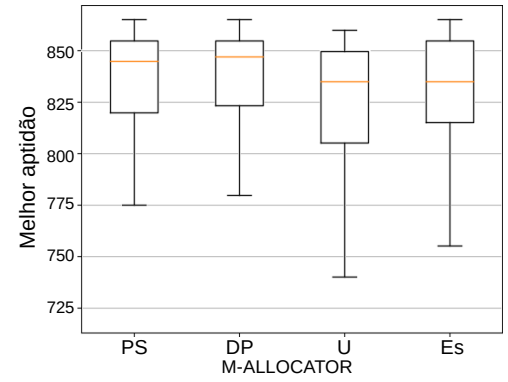

(b) Aptidão

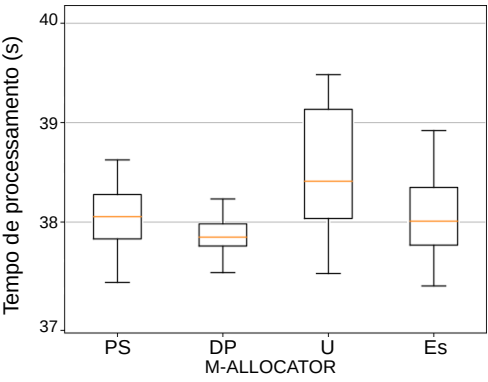

(c) Tempo

Figura 4. Impacto de desempenho com a variação dos métodos de reprodução.

Em relação ao método de mutação, observa-se que há uma convergência mais rápida do M-ALLOCATOR ao usar o método de EM, aproximando-se da solução exata à medida que a quantidade de geração aumenta. Além disso, vale frisar que não há uma diferença na métrica aptidão e tempo de processamento ao usar os métodos EM e I.

Como observação da definição do problema, destaca-se que dentre as restrições apresentadas na modelagem do problema, observou-se que a Restrição 4 gerou mais impacto nos resultados obtidos, independente dos métodos utilizados para avaliação. Isso ocorre devido à restrição impor que os nós de acesso sejam distribuídos em clusters. Isso aumenta a interferência co-canal entre as células, além de reduzir a área de cobertura do 
sistema e o número de clientes atendidos. Entretanto, destaca-se que o E-ALLOCATOR é adequado quando há baixa carga de trabalho, enquanto o M-ALLOCATOR é adequado para os cenários com alta carga de trabalho. Ainda, M-ALLOCATOR fornece soluções quase ótimas dentro do tempo computacional adequado para todas as instâncias do problema, como apresentado na Tabela 4.

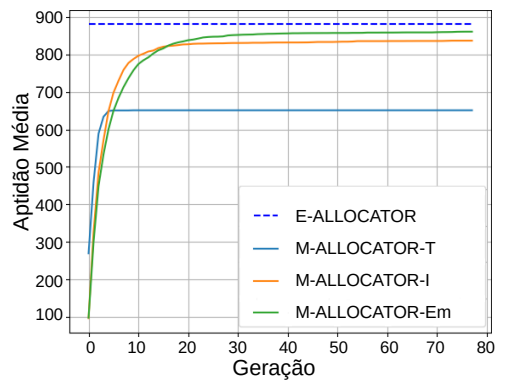

(a) Convergência

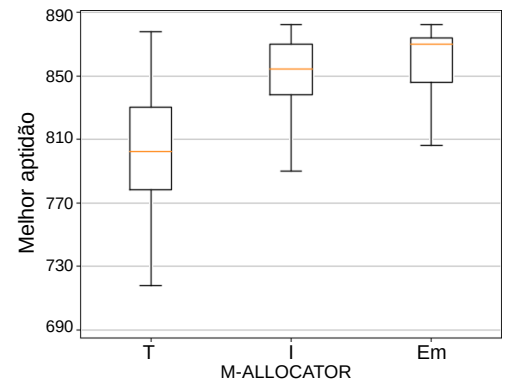

(b) Aptidão

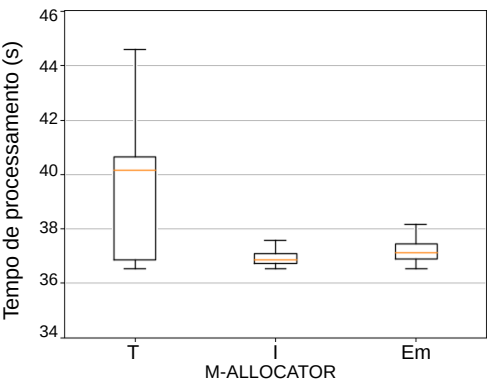

(c) Tempo

Figura 5. Impacto de desempenho com a variação dos métodos de mutação.

Tabela 4. Desempenho das soluções otimizadas (Média \pm IC de 95\%).

\begin{tabular}{c|c|c|c|c|c}
\hline \multirow{2}{*}{ Cenário } & \multicolumn{2}{|c|}{ E-ALLOCATOR } & \multicolumn{2}{c}{ M-ALLOCATOR-EE/DP/Em } \\
\cline { 2 - 6 } & Tempo (s) & Objetivo & Tempo (s) & Objetivo & PRD \\
\hline $200 \times 50$ & $0,62 \pm 0,0304$ & $876 \pm 0$ & $38,09 \pm 0,2607$ & $817,81 \pm 3,8728$ & $7,12 \pm 0,51$ \\
\hline $800 \times 400$ & $>3$ dias & - & $587,39 \pm 9,2118$ & $1746,83 \pm 14,7703$ & - \\
\hline $3000 \times 400$ & $>7$ dias & - & $1497,21 \pm 23,4666$ & $6100,40 \pm 118,7256$ & - \\
\hline
\end{tabular}

\section{Conclusões e Trabalhos Futuros}

Para resolver o problema de localização dos nós de acesso do CCOp Mv, este artigo propôs um modelo analítico baseado no PLIM, o qual foi utilizado como base para a modelagem de duas soluções otimizadas: (i) E-ALLOCATOR; e (ii) M-ALLOCATOR. Ambas soluções além de cobrir o maior número de pontos de demanda com o menor número de nós de acesso, tem como objetivo prover uma maior área de apoio nas operações que necessitam de comunicação crítica.

Para validar a viabilidade das soluções propostas foi modelado um cenário com base na tragédia ocorrida em janeiro de 2019 em Brumadinho-MG. Os resultados mostraram que o E-ALLOCATOR é adequado quando há baixa carga de trabalho, enquanto o M-ALLOCATOR é adequado para os cenários com alta carga de trabalho. Ainda, os resultados demonstram que o tempo de computação do E-ALLOCATOR aumenta significativamente com o tamanho do problema, enquanto o M-ALLOCATOR fornece soluções quase ótimas dentro do tempo computacional adequado para todas as instâncias.

Como trabalho futuro, planeja-se desenvolver um mecanismo de clusterização dos pontos de demanda mediante a distância euclidiana de maneira a agrupar os pontos próximos durante a etapa de pré-processamento. Com isso, as matrizes a serem processadas na etapa de otimização terão dimensões menores e demandarão menor poder computacional para chegar às soluções. 


\section{Referências}

Does outdoor antenna increase the speed of lte? https://www.dipolnet.com/ does_outdoor_antenna_increase_the_speed_of_lte_bib532. htm. acessado em 18/06/2021.

Alizadeh, R., Nishi, T., Bagherinejad, J., and Bashiri, M. (2021). Multi-period maximal covering location problem with capacitated facilities and modules for natural disaster relief services. Applied Sciences, 11(1):397.

Atta, S., Mahapatra, P. R. S., and Mukhopadhyay, A. (2018). Solving maximal covering location problem using genetic algorithm with local refinement. Soft Computing, 22(12):3891-3906.

Bagherinejad, J. and Shoeib, M. (2018). Dynamic capacitated maximal covering location problem by considering dynamic capacity. International Journal of Industrial Engineering Computations, 9(2):249-264.

Church, R. and ReVelle, C. (1974). The maximal covering location problem. In Papers of the regional science association, volume 32, pages 101-118. Springer-Verlag.

Costa, V. O. (2016). Alocação de antenas para rede celular de 4g utilizando algoritmos meméticos. Dissertação de Mestrado.

Farahani, R. Z., Asgari, N., Heidari, N., Hosseininia, M., and Goh, M. (2012). Covering problems in facility location: A review. Computers \& Industrial Engineering, 62(1):368-407.

Gazani, M. and Niaki, S. (2021). The capacitated maximal covering location problem with heterogeneous facilities and vehicles and different setup costs: An effective heuristic approach. International Journal of Industrial Engineering Computations, 12(1):79_ 90 .

Mathar, R. and Niessen, T. (2000). Optimum positioning of base stations for cellular radio networks. Wireless Networks, 6(6):421-428.

Mehboob, U., Qadir, J., Ali, S., and Vasilakos, A. (2016). Genetic algorithms in wireless networking: techniques, applications, and issues. Soft Computing, 20(6):2467-2501.

Rappaport, T. S. et al. (1996). Wireless communications: principles and practice, volume 2. Prentice Hall PTR New Jersey.

Seda, P., Seda, M., and Hosek, J. (2020). On mathematical modelling of automated coverage optimization in wireless $5 \mathrm{~g}$ and beyond deployments. Applied Sciences, 10(24):8853.

Seybold, J. S. (2005). Introduction to RF propagation. John Wiley \& Sons.

Yang, P., Xiao, Y., Zhang, Y., Zhou, S., Yang, J., and Xu, Y. (2020). The continuous maximal covering location problem in large-scale natural disaster rescue scenes. Computers Industrial Engineering, 146:106608. 